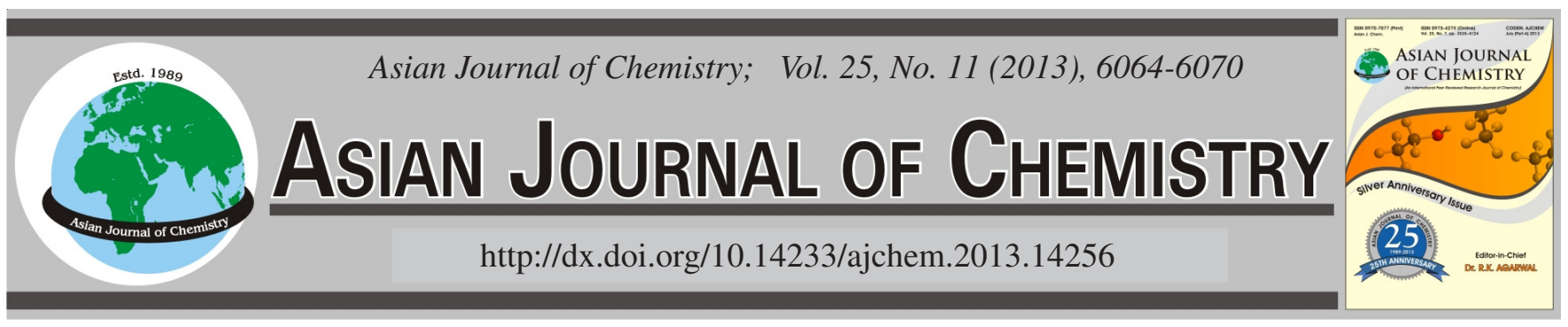

\title{
Comparative Study of the Antioxidant Activities of Water- Soluble Vitamin E Analogues: Trolox and IRFI
}

\author{
Mahdi Rafiei ${ }^{1}$, Abolfazl Barzegar ${ }^{2,3, *}$ and Asadollah Asadi ${ }^{4}$
}

${ }^{1}$ Reference Health Laboratory of Health Minstry, Tehran, Iran

${ }^{2}$ Research Institute for Fundamental Sciences, University of Tabriz, Tabriz, Iran

${ }^{3}$ Faculty of Advanced Medical Sciences, Tabriz University of Medical Sciences, Tabriz, Iran

${ }^{4}$ Department of Biology, Faculty of Science, University of Mohaghegh Ardabili, Ardabil, Iran

*Corresponding author: Fax: +98 411 3294113; Tel: +98 411 3294114; E-mail: barzegar@ tabrizu.ac.ir

(Received: 30 June 2012;

Accepted: 29 April 2013)

AJC-13402

The potent antioxidant activity of a novel synthetic-hydrophilic analogue of vitamin E (IRFI) was comparatively studied with the known
water-soluble vitamin E derivative (Trolox). Evaluation of molecular properties indicated the higher hydrophilic property and water
solubility of IRFI than Trolox. But Trolox is more planar than IRFI analogue. The spectroscopic behaviour of IRFI and Trolox was almost
the same with the maximum absorbance of 295 and $300 \mathrm{~nm}$ respectively. Cellular antioxidant and antitoxicity assays showed that both
IRFI and Trolox freely permeate across cellular membranes, enabling them to be an effective suppressor of intracellular ROS and to
protect cells against toxicity. Reduction of ferric ion was efficient for IRFI as well as Trolox in phosphate buffer solution. Data indicated
that every one of IRFI and Trolox molecules enables to reduce two galvinoxyl free radicals not only in polar protic ethanol but also in
polar aprotic acetonitrile solvents. Although $\mathrm{H}$ elimination process in phenolic hydroxyls of IRFI and TxOH is more feasible to free
radical reduction than $\alpha$-tocopherol but the antioxidant activity of TxOH and IRFI is different that mainly via $\mathrm{H}$ atom and electron transfer
mechanisms respectively. Interestingly, $\alpha$-tocopherol locates in the borderline for $\mathrm{H}$ atom and electron transfer mechanisms of radical
suppressing.

Key Words: IRFI, Trolox, ROS, Antioxidant, Reducing power, Free radical, DFT, Antioxidant.

\section{INTRODUCTION}

Vitamin E, a generic term, refers to a group of eight fatsoluble compounds that include both tocopherols $(\alpha-, \beta-, \gamma-$ and $\delta$-) and tocotrienols $(\alpha-, \beta-, \gamma \text { - and } \delta-)^{1}$. $\alpha$-Tocopherol is the most plentiful and the most biologically active form of vitamin $\mathrm{E}$. It is the only form of vitamin $\mathrm{E}$ that is actively maintained in the human body. The action of $\alpha$-tocopherol as a powerful antioxidant against free radicals has been the subject of extensive studies in the last three decades ${ }^{2,3}$. It represents the major lipid-soluble antioxidant activity to prevent the peroxidation of membrane phospholipids and to avoid cell membrane damage caused by an excess of free radicals ${ }^{3}$. Free radicals are formed primarily in the body during normal breathing and metabolism (chemical reactions in the body). Although human must breathe oxygen to stay alive, oxygen is a risky substance inside the body because it can make molecules overly reactive known oxygen reactive species (ROS) ${ }^{4,5}$. When oxygen-containing molecules become too reactive, they can start damaging the cell structures around them. In chemistry, this imbalanced situation involving oxygen is called oxidative stress. However, antioxidant molecules help to prevent oxidative stress. The fat-soluble $\alpha$-tocopherol, is uniquely suited to intercept free radicals and thus prevent a chain reaction of lipid destruction ${ }^{3}$. But, $\alpha$-tocopherol is insoluble in water and unstable that its colour gradually changes into dark brown after exposure to air or light ${ }^{1}$. Hence, water insolubility, limits the $\alpha$-tocopherol's ROS scavenging potency in aqueous medium such as, serum, plasma, extracellular fluid and especially inside of the cells. Excess formation of ROS through inside of cells by various enzymatic and nonenzymatic processes has been associated with the oxidation of $\mathrm{DNA}^{6}$. Trolox $(\mathrm{TxOH})$, the well known water-soluble analogue of $\alpha$-tocopherol, is usually used instead of $\alpha$-tocopherol as a standard antioxidant because of having both water-solubility and high stability ${ }^{7}$. It is a watersoluble derivative of vitamin $\mathrm{E}$ with potent antioxidant, used in biological or biochemical applications to reduce oxidative stress or damage. $\mathrm{TxOH}$ is commonly used as a standard or positive control in antioxidant assays ${ }^{8}$. The other novel watersoluble analogue of vitamin E is IRFI005 (2-[5-hydroxy-4,6,7trimethyl-2,3-dihydro-1-benzofuran-2-yl] acetic acid) derived from raxofelast. It has been designed with the aim of maximizing 
the antioxidant potency of phenols chemically related to $\alpha$ tocopherol $^{9,10}$. Structural formulae of $\alpha$-tocopherol, Trolox and IRFI depicted in Fig. 1. In the present study, the comparative antioxidant activities of two water-soluble vitamin $\mathrm{E}$ analogues (IRFI and TxOH), have been evaluated using different methods. Data demonstrated that, the new hydrophilic vitamin E-like antioxidant, IRFI, has high antioxidant activity as well as TxOH and can be used instead of TxOH thus IRFI as a positive control in antioxidant assays or therapeutic strategies for inhibition of oxidative stress.

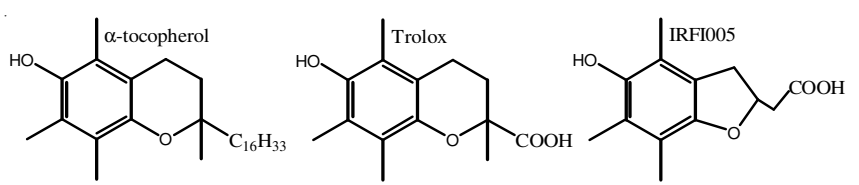

Fig. 1. Structural formulae of $\alpha$-tocopherol, TxOH and IRFI

\section{EXPERIMENTAL}

The analytical grade of reagents including Trolox $(\mathrm{TxOH})$, galvinoxyl radical and trichloroacetic acid (TCA), cumene hydroperoxide were purchased from Sigma. IRFI (IRFI005) was gift from Biomedica Foscama (Ferentino, Italy). Ethanol, acetonitrile, potassium ferricyanide and ferric chloride were purchased from Merck. Dichlorodihydrofluorescein diacetate (DCFH2-DA) was obtained from molecular probes (Eugene, OR). Dulbecco's modified Eagle's medium (DMEM), antibiotics and sterile plasticware for cell culture were from flow laboratory (Irvine, UK).

Galvinoxyl free radical scavenging assay: Free radical scavenging activity of IRFI and $\mathrm{TxOH}$ has been evaluated using galvinoxyl method. $10 \mu \mathrm{M}$ solution of galvinoxyl was thoroughly mixed with different concentrations of IRFI and $\mathrm{TxOH}$ antioxidants. The molar ratios for both of antioxidants relative to galvinoxyl radical were 1:10, $1: 4$ and 1:2 in solution. The optical behaviour of galvinoxyl in the presence of antioxidants was studied for different incubated times.

Ferric ion reduction: Electron-transfer potency of IRFI and $\mathrm{TxOH}$ was evaluated based on the method of ferric ion (iron in the +3 oxidation state, $\mathrm{Fe}^{3+}$ ) reduction approach. The process by which the ferric ion reduces into the +2 oxidation ferrous ion state $\left(\mathrm{Fe}^{2+}\right)$ is known reducing power method ${ }^{11}$. Briefly, potassium ferricyanide (1\%) was shortly incubated with different concentrations of antioxidants for $0.5 \mathrm{~h}$ at $50{ }^{\circ} \mathrm{C}$ in phosphate buffered saline (PBS). After adding trichloroacetic acid $(10 \%)$ and $\mathrm{FeCl}_{3}(0.1 \%)$ the absorbance at $700 \mathrm{~nm}$ was recorded as reducing power of antioxidants in phosphate buffered saline solution.

Intracellular oxidative free radicals: DCF method was applied for the intracellular ROS evaluation that we have explained recently ${ }^{12}$. Briefly, L-6 cells were incubated with dichlorodihydrofluoroscein diacetate $\left(\mathrm{DCFH}_{2}-\mathrm{DA}\right)$ in phosphate buffered saline. Then intracellular ROS was induced by addition of highly proxidant cumene hydroperoxide compound that causes oxidation of $\mathrm{DCFH}_{2}$. This process results in appearance of fluorescent DCF compound. The amount of intracellular DCF was easily followed by excitation at $498 \mathrm{~nm}$ and emission $530 \mathrm{~nm}$ using fluorimeter ${ }^{13}$. The concentration of antioxidants in which the fluorescence intensity decreased to $50 \%$ of its initial value used as the $\mathrm{IC}_{50}$ value for each sample. Experiments were done in triplicate and the mean value was recorded.

Cell viability assay: Cell viability assayed in order to evaluate the antitoxic capacity of TxOH and IRFI antioxidants. Methylthiazole tetrazolium method used for assaying the viable cells in the presence and absence of antioxidants ${ }^{12}$. The viability of cells was quantified by the conversion of yellow methylthiazole tetrazolium to purple methylthiazole tetrazolium formazan by the mitochondrial dehydrogenases of living cells. A decrease in viable cell numbers results in a decrease purple methylthiazole tetrazolium formazan absorbance at 560 $\mathrm{nm}$. The percentages of cell viability were normalized and calculated with the following equation:

$$
\text { Cell Viability }(\%)=\left(\mathrm{OD}_{\text {test }} / \mathrm{OD}_{\text {control }}\right) \times 100
$$

Chemical computations studies: Quantum chemical computations were aimed to determine the molecular geometry and energetic properties associated with the antioxidant parameters of the molecule. For this purpose, density-functional-theory (DFT) method applied to calculate ionization potential (IP) and bond dissociation enthalpy (BDE) ${ }^{14}$. The bond dissociation enthalpy and ionization potential values for $\mathrm{TxOH}$ and IRFI were calculated at two computational levels as (RO)B3LYP/6-311+G(2d,2p)//AM1/AM1 and (U)B3LYP/6-31G(d)//AM1/AM1 ${ }^{15}$. The molecular geometries were first optimized by the molecular-mechanic MM+ method and followed by the semiempirical quantumchemical method AM1 by determining vibrational frequen$\operatorname{cies}^{15}$. The molecular energy consists of (RO)B3LYP/6$311+\mathrm{G}(2 \mathrm{~d}, 2 \mathrm{p})$-calculated single-point electronic energies (SPEs) and AM1-calculated zero-point vibration energy (ZPVE) scaled by a factor of $0.973^{14,16}$. ROB3LYP refers to restricted open-shell calculation that used for the radical generated after $\mathrm{H}$-abstraction. Bond dissociation enthalpy values calculated with $\mathrm{H}_{\mathrm{r}}+\mathrm{H}_{\mathrm{h}}-\mathrm{H}_{\mathrm{p}}$, in which, $\mathrm{H}_{\mathrm{r}}$ is the enthalpy of radical generated after $\mathrm{H}$-abstraction reaction, $\mathrm{H}_{\mathrm{h}}$ is the enthalpy of the hydrogen-atom, 0.49765 Hartree and $\mathrm{H}_{\mathrm{p}}$ is the enthalpy of the parent molecule.

The ionization potential was defined as $\mathrm{E}_{\mathrm{c}}-\mathrm{E}_{\mathrm{p}}$, where $\mathrm{E}_{\mathrm{c}}$ is the energy for the cation radical and $E_{p}$ is the energy for the parent molecule. During the calculations of ionization potentials, the molecular energy in the gas-phase consists of (U)B3LYP/6-31G(d)-calculated single-point electronic and AM1-calculated ZPVE (scaled by a factor of 0.973$)^{16}$. UB3LYP refers to the unrestricted Open-Shell calculation that used for the radical generated after electron-abstraction (cation radicals). The quantum-chemical calculations were accomplished by the Gaussian 98 program $^{17}$.

\section{RESULTS AND DISCUSSION}

Optical absorption behaviour of IRFI and Trolox: The structures of IRFI005 (IRFI) and Trolox (TxOH), present an aromatic ring, which is responsible for the characteristic of UV-visible absorption. The spectroscopic behaviour of IRFI and $\mathrm{TxOH}$ are almost the same having maximum absorbance at 295 and $300 \mathrm{~nm}$ in ethanol respectively (Fig. 2). Fortunately they have no absorbance band in the ranges $\lambda>310 \mathrm{~nm}$. It causes feasible evaluation of IRFI and $\mathrm{TxOH}$ antioxidant activity to scavenge free radical using different stable free 
radicals such as galvinoxyl radicals by UV-visible spectrophotometer.
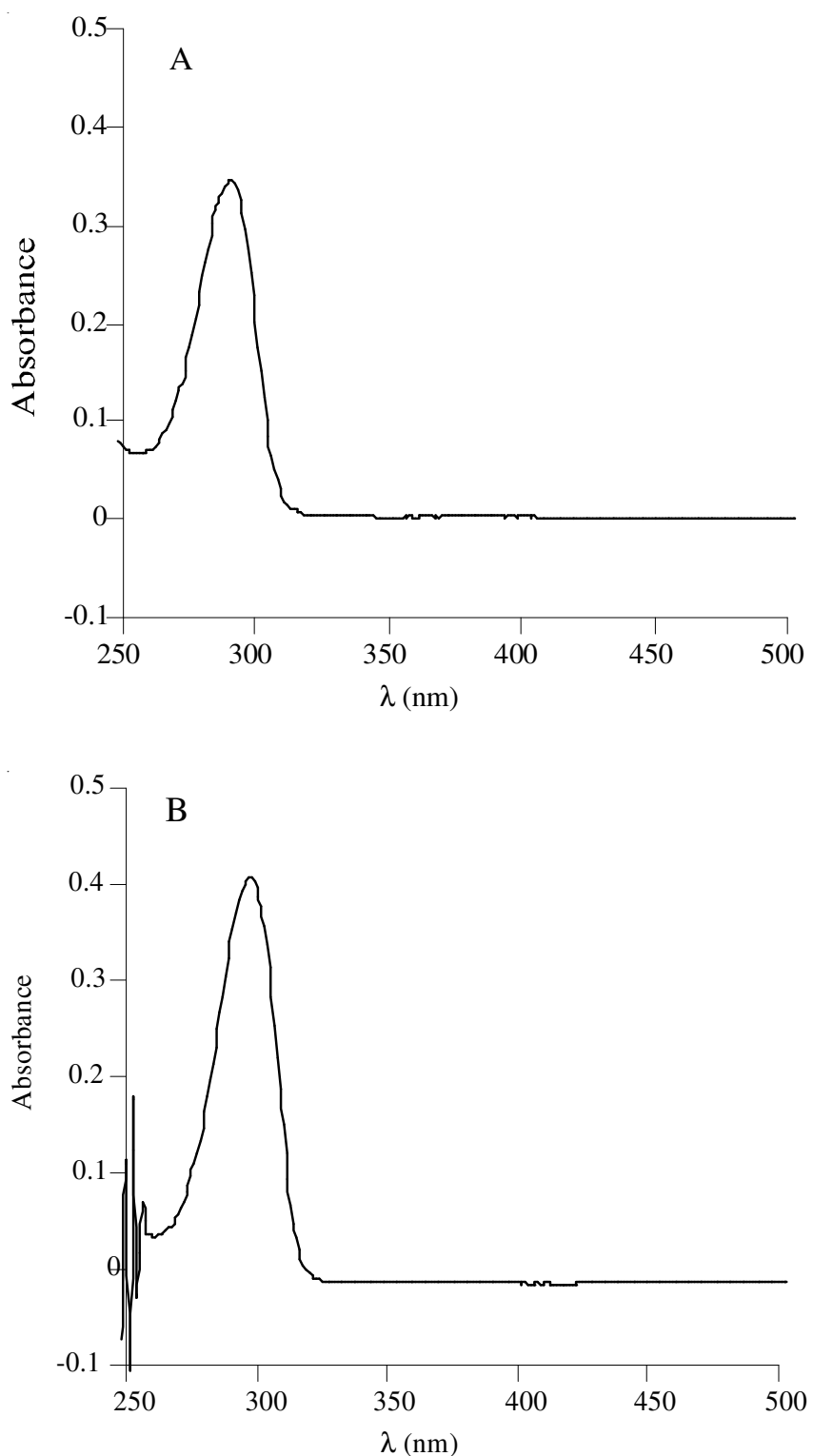

Fig. 2. Optical absorption spectra of IRFI and TxOH in ethanol. Panels A and B indicate the spectra for $100 \mu \mathrm{M}$ of IRFI and Trolox in ethanol respectively

Galvinoxyl free radicals $(\mathbf{G x})$ reduction: This assay is based on the ability of the stable galvinoxyl radicals, Gx, to react with hydrogen and/or electron donors antioxidants. Reduction of Gx is well known method for studying electron and proton donation antioxidant activities ${ }^{18}$. It is possible to study reduction of free radicals by antioxidants using UV-visible spectroscopy. Fig. 3 indicates that Gx radicals exhibit optical absorbance in the visible range, with $\lambda_{\max }$ at $430 \mathrm{~nm}$ but reduction to $\mathrm{GxH}$ causes the absorbance maximum shifted to $400 \mathrm{~nm}$. In this assay, a solution of Gx radical, which has a deep purple colour, is decolourized after reduction with IRFI and $\mathrm{TxOH}$ accordingly;

$\mathrm{Gx}+$ IRFIOH $(\mathrm{TxOH}) \rightarrow \mathrm{GxH}+$ IRFIO^ $\left(\mathrm{TxO}^{\wedge}\right)$

Data from Fig. 3 refer that $10 \mu \mathrm{M}$ of Gx radicals completely disappeared by $5 \mu \mathrm{M}$ of IRFI and TxOH in ethanol separately. It means two electrons or two $\mathrm{H}$-atoms being donated from the IRFI and TxOH enable complete reduction of Gx radicals. To confirm these results, we have evaluated their free radical reduction potency in two different solutions including polar protic, ethanol and polar aprotic, acetonitrile solvents. Fig. 4 shows that with increasing concentration of IRFI and $\mathrm{TxOH}$, the amount of Gx radicals decreased. A decrease was observed almost the same not only for both of IRFI and TxOH in ethanol but also the same in acetonitrile as well. These data confirm that polar protic ethanol and polar aprotic acetonitrile have no effect on the Gx radicals reduction potency of IRFI and TxOH. Moreover, Fig. 4 clearly demonstrates that both of IRFI and $\mathrm{TxOH}$ transfer two electrons or $\mathrm{H}$-atoms to scavenge Gxi radicals.
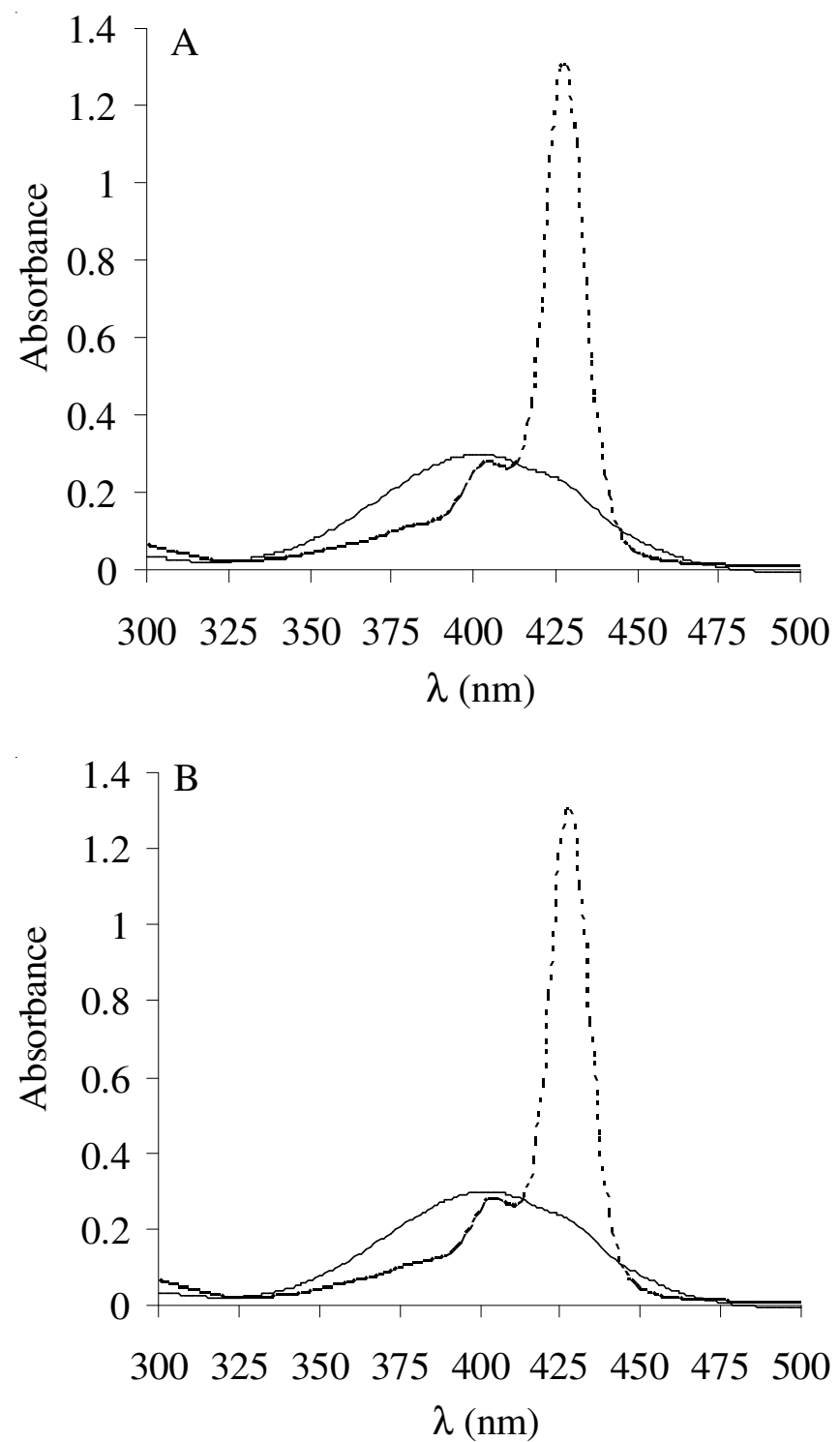

Fig. 3. Optical absorption spectra of galvinoxyl radical $\left(\mathrm{Gx}^{\wedge}\right)$ in ethanol. $10 \mu \mathrm{M}$ of $\mathrm{Gx}^{2}$ in the presence of $5 \mu \mathrm{M}$ IRFI (Panel A) and $5 \mu \mathrm{M}$ Trolox (Panel B) in Ethanol. The radical Gx and reduced galvinoxyl $(\mathrm{GxH})$ spectra depicted with continuous and discontinuous lines respectively. The radical Gx has $\lambda_{\max }$ at $430 \mathrm{~nm}$ but reduction to GxH causes the absorbance $\lambda \max$ shifted to $400 \mathrm{~nm}$. Because of the interference in absorbance of reduced product of galvinoxyl $\mathrm{GxH}$ with Gx' radical, the absorbance value at $400 \mathrm{~nm}$ cannot come down to zero 

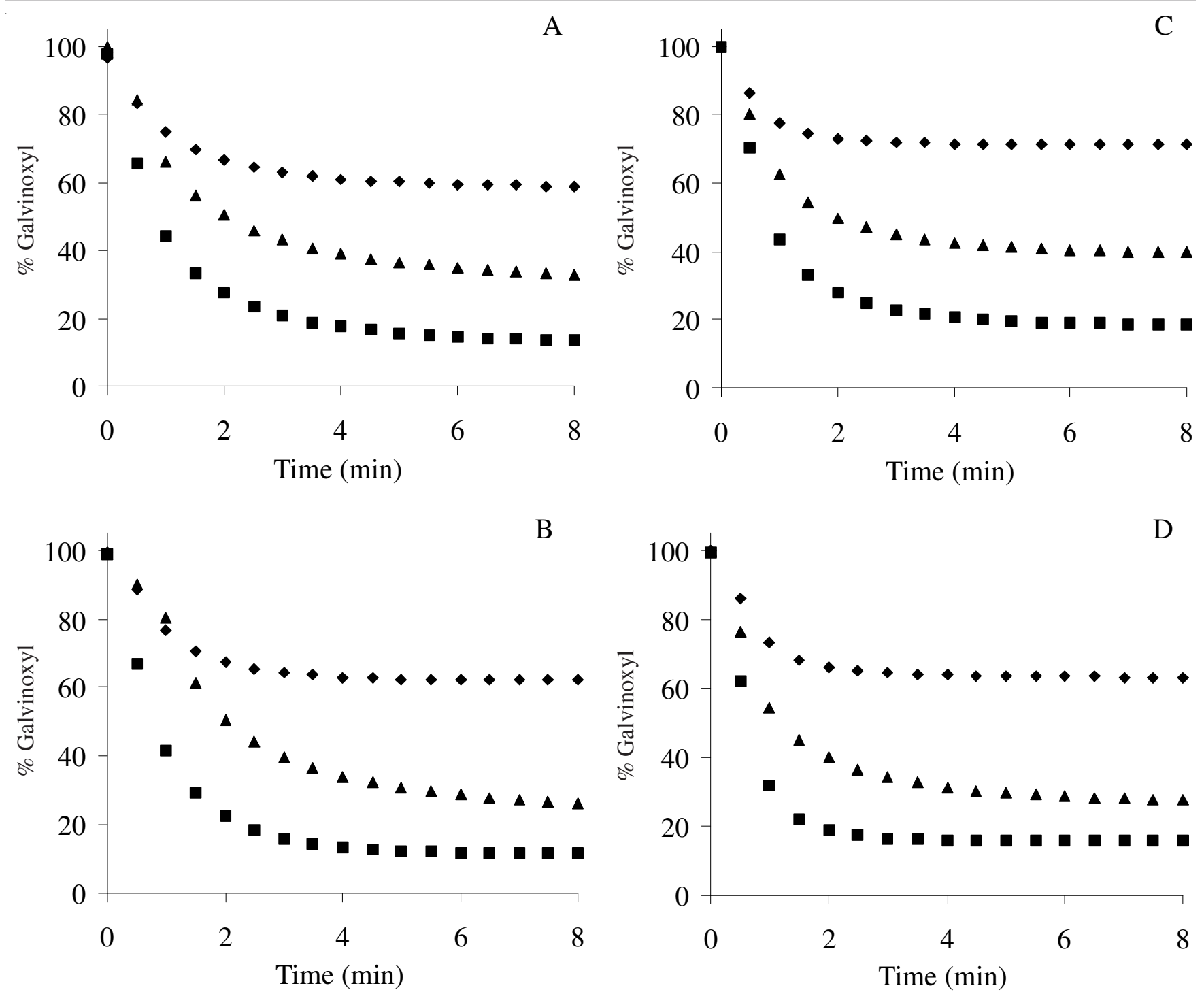

Fig. 4. Kinetic galvinoxyl free radical (Gox) scavenging activity of IRFI and $\mathrm{TxOH}$ in ethanol, $\mathrm{C}_{2} \mathrm{H}_{5}-\mathrm{OH}$, (panel A and C) as well as acetonitrile, $\mathrm{CH}_{3}-\mathrm{C}=\mathrm{N}$, (panels B and D). In this experiment, $990 \mu \mathrm{L}$ of $10 \mu \mathrm{M}$ solution of galvinoxyl was thoroughly mixed with $10 \mu \mathrm{L}$ volume of IRFI and TxOH separately. Panels A and B denote the Gox scavenging activity assays using different concentrations of IRFI whereas panels C and D refer in the presence of TxOH. Different molar ratios of IRFI and TxOH added to the radical samples; $0.1(\bullet), 0.25(\boldsymbol{\Delta})$ and $0.50(\boldsymbol{\square})$. In the molar ratios of 0.50 , Gox radicals completely reduced in both of ethanol and acetonitrile solution

Ferric ion reducing: Electron-transfer reaction of IRFI and TxOH has been focused on Fe(III) reduction to Fe(II) in aqueous solution that can be a significant indicator of the antioxidant activity. The reduction of $\mathrm{Fe}(\mathrm{III})$ in presence of the IRFI and TxOH presented in Fig. 5. Data demonstrate that IRFI has similar to $\mathrm{TxOH}$ equivalent reducing capacity effects. It means, both IRFI and $\mathrm{TxOH}$ have electron-transfer properties which are active in polar phosphate buffer medium.

Intracellular ROS scavenging: L-6 myoblasts were applied as an in vitro cell model to investigate the intracellular ROS scavenging potency of IRFI and TxOH based on the DCF method $^{12}$. This method was applied to detect levels of intracellular ROS compounds in myoblasts treated by oxidative stressor cumene hydroperoxide. Cumene hydroperoxide is one of the oxidizing agents used as an intracellular source of reactive oxygen intermediates ${ }^{12}$. Fig. 6 demonstrates that both IRFI and $\mathrm{TxOH}$ intercept intracellular peroxide-forming radicals result in preventing the DCF fluorescence intensity. Cumene hydroperoxide easily permeates through the cell membrane which, in the absence of IRFI and $\mathrm{TxOH}$, causes DCF oxidation and its fluorescence intensity increment. Whereas, IRFI and TxOH suppressed the ROS generation inside the cells. Moreover, to examine the ability of IRFI and $\mathrm{TxOH}$ to reduce intracellular $\mathrm{ROS}$ we used $\mathrm{IC}_{50}$ reports. $\mathrm{IC}_{50}$ is inversely related to the antioxidant capacity of a compound, as it expresses the amount of antioxidant needed to decrease the ROS concentrations by $50 \%$. The lower $\mathrm{IC}_{50}$, the higher the biological antioxidant activity of a compound is. Table- 1 indicated the high efficiency of IRFI and TxOH to reduce intracellular ROS. Intracellular ROS scavenging capability of IRFI is comparable and/or more effective than TxOH. Efficiently suppressing intracellular ROS production, demonstrates that both IRFI and TxOH compounds diffuse through the cell membrane into the cells and act to prevent of $\mathrm{DCFH}_{2}$ conversion to fluorescent DCF. 


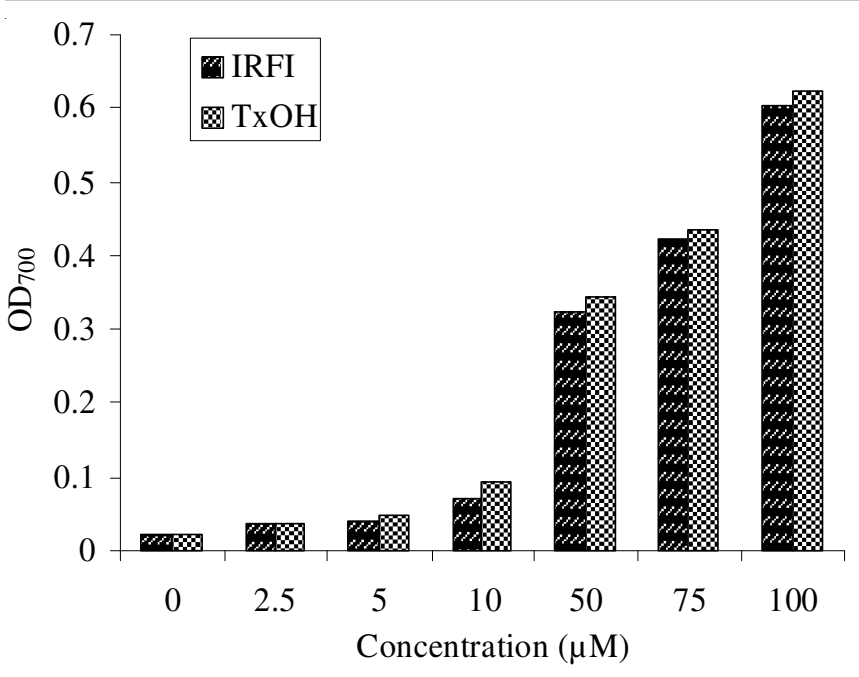

Fig. 5. Electron donating capacity of IRFI and Trolox (TxOH) to ferric iron $\left(\mathrm{Fe}^{3+}\right)$ reduction. The absorbance at $700 \mathrm{~nm}$ was recorded as a function of IRFI and TxOH concentrations in phosphate buffered saline to monitor the reduction of ferric ion to ferrous ion. Increased the concentrations of both IRFI and $\mathrm{TxOH}$ led to an increase in ferric ion reduction

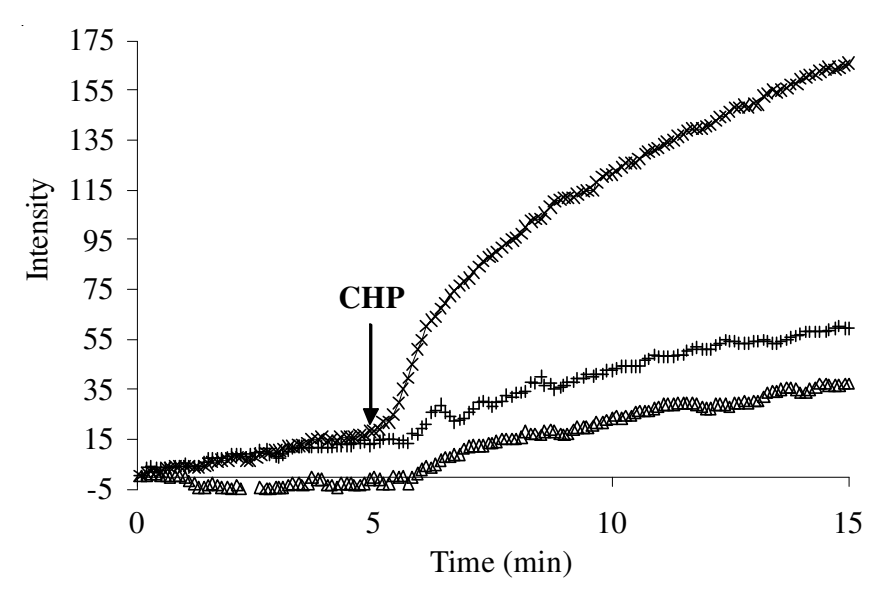

Fig. 6. Intracellular ROS determination by DCF method. Samples were first incubated $(30 \mathrm{~min}$ ) with $0.0(\times), 1.0 \mu \mathrm{M} \mathrm{TxOH}(+)$ and $1.0 \mu \mathrm{M}$ IRFI $(\Delta)$. The changes of fluorescence intensity were monitored for the following intracellular ROS. The fluorescence behaviour of the samples was monitored by excitation at $498 \mathrm{~nm}$ and emission $530 \mathrm{~nm}$. Samples were induced to produce intracellular ROS and radical generator by adding the ROS stimulating agent of cumene hydroperoxide (CHP) that was mentioned by arrow

\section{TABLE-1}

INTRACELLULAR ROS SCAVENGING ACTIVITY OF TxOH AND IRFI. THE CONCENTRATION OF ANTIOXIDANT THAT CAUSED THE INITIAL ROS TO DECREASE $50 \%$ INDICATED AS IC 50

\begin{tabular}{lc}
\hline Compounds & $\mathrm{IC}_{50}(\mu \mathrm{M})$ \\
\hline IRFI & $0.40( \pm 0.1)$ \\
TxOH & $0.50( \pm 0.1)$ \\
\hline
\end{tabular}

Cellular antitoxicity assay: Antitoxic property of IRFI and $\mathrm{TxOH}$, based on protection of viable myoblasts against cytotoxic and lethal effects of cumene hydroperoxide, was evaluated using methylthiazole tetrazolium assay. Cumene hydroperoxide has been frequently used as a model compound to study both the organic hydroperoxides and the mechanisms driving oxidative cell injury in mammalian cells ${ }^{19}$. For this purpose, cells first were treated with $5 \mu \mathrm{M}$ concentrations of
IRFI and TxOH then stimulated by cumene hydroperoxide as a strong toxic organic hydroperoxide molecule. Cumene hydroperoxide was observed to be a highly toxic compound for cells but IRFI and TxOH help to block the toxic effect of cumene hydroperoxide (Fig. 7). These data confirm the abovementioned findings indicated that IRFI and $\mathrm{TxOH}$ easily diffuse through the cell membrane into the cells and blocks producing toxic ROS reagents. The concentration of $5 \mu \mathrm{M}$ of IRFI blocks the toxic effect of cumene hydroperoxide (Fig. 7). The results from intracellular ROS and methylthiazole tetrazolium assays are consistent and show highly radical chain breaking potency of IRFI and $\mathrm{TxOH}$. In order to compare the mechanism of the high efficient antioxidant activity of IRFI and $\mathrm{TxOH}$ the additional following computations were carried out.

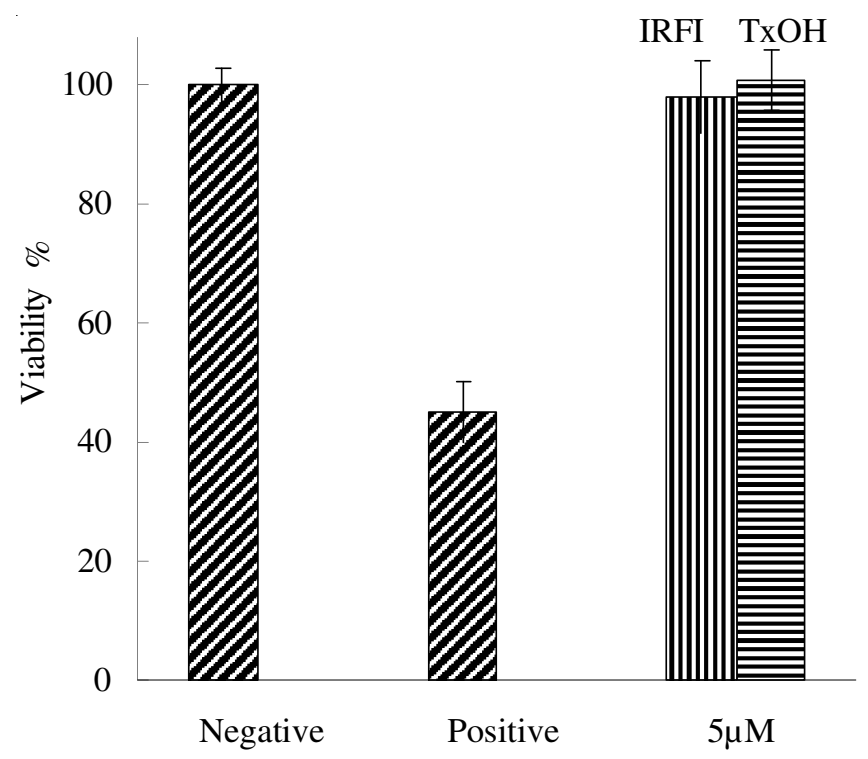

Fig. 7. Cell viability against cytotoxic effect of CHP using MTT assays. Separately, cells were treated with $5 \mu \mathrm{M}$ IRFI and $\mathrm{TxOH}$, then were induced with $5 \mu \mathrm{L}(1: 100) \mathrm{CHP}$. Negative control refers without any addition of antioxidant and positive control denoting only presence of CHP. It was assayed two times with different plates in different days. No detectable toxic effect on cell viability has been recorded for IRFI and $\mathrm{TxOH}$

DFT calculations: DFT calculations have been carried out to elucidate the role of hydrogen atom transfer (HAT) and/ or electron transfer (ET) mechanism to radical scavenge by IRFI and TxOH. Two main parameters for evaluating the mechanism of antioxidant capacity of a molecule are bonddissociation enthalpy (BDE) and ionization potential. Low bond dissociation enthalpy value refers to feasible $\mathrm{H}$ atom abstraction which is important in determining a good antioxidant activity ${ }^{11}$. $\mathrm{H}$ elimination process for reduction of proxy radicals $\left(\mathrm{RO}_{2}\right)$ by phenolic hydroxyl group of IRFI and TxOH can be raised as eqn. 2;

$\mathrm{RO}_{2}{ }^{+}+$IRFIOH (TxOH) $\rightarrow \mathrm{ROOH}+$ IRFIO (TxO) $^{\wedge}$ (2)

Based on the data from Table-2, $\mathrm{H}$ abstraction process in phenolic hydroxyl of IRFI is more feasible than $\mathrm{TxOH}$ and both of them are favourable and more labile than $\alpha$-tocopherol. Already the bond dissociation enthalpy value for $\alpha$-tocopherol was reported $78.93 \pm 0.39 \mathrm{kcal} / \mathrm{mol}^{20}$ and $76 \mathrm{kcal} / \mathrm{mol}^{21}$ using different methods. Herein, we used full-basis DFT methodology 
TABLE-2

ELECTRONIC ENERGIES AND H ATOM BOND DISSOCIATION ENTHALPIES (BDEs, kcal/mol) OF TxOH, IRFI AND $\alpha$-TOCOPHEROL. PARAMETERS HAVE BEEN COMPUTED USING B3LYP/6-311+G(2d, 2p)//AM1 CALCULATIONS

\begin{tabular}{lccccc}
\hline Compounds & $\mathrm{SPE}_{\mathrm{p}}$ & $\mathrm{ZPVE}_{\mathrm{p}}$ & $\mathrm{SPE}_{\mathrm{r}}$ & $\mathrm{ZPVE}_{\mathrm{r}}$ & $\mathrm{BDE}$ \\
\hline TxOH & -845.5055599 & 0.323805 & -844.8732957 & 0.310152 & 76.14 \\
IRFI & -806.1823663 & 0.294373 & -805.5519216 & 0.280787 & 75.04 \\
$\alpha$-Tocopherol & -1089.4423908 & 0.635305 & -1088.8090438 & 0.621762 & 76.90
\end{tabular}

$\mathrm{SPE}_{\mathrm{p}}$, Single-point energies of parent molecule. $\mathrm{ZPVE}_{\mathrm{p}}$, Zero-point vibrational energies of parent molecule. SPE $\mathrm{r}_{\mathrm{r}}$, Single-point energies of radical generated after $\mathrm{H}$-abstraction. $\mathrm{ZPVE}_{\mathrm{r}}$, Zero-point vibrational energies radical generated after $\mathrm{H}$-abstraction

TABLE-3

ELECTRONIC ENERGIES AND IONIZATION POTENTIALS (IPs, $\mathrm{kcal} / \mathrm{mol}$ ) OF TxOH, IRFI AND $\alpha$-TOCOPHEROL. PARAMETERS HAVE BEEN COMPUTED USING B3LYP/6-31G(d)//AM1 CALCULATIONS

\begin{tabular}{lccccc}
\hline Compounds & $\mathrm{SPE}_{\mathrm{p}}$ & $\mathrm{ZPVE}_{\mathrm{p}}$ & $\mathrm{SPE}_{\mathrm{r}}$ & $\mathrm{ZPVE}_{\mathrm{r}}$ & $\mathrm{IP}$ \\
\hline TxOH & -845.231332 & 0.323805 & -844.986911 & 0.321728 & 152.10 \\
IRFI & -805.911118 & 0.294373 & -805.681395 & 0.292241 & 142.85 \\
$\alpha$-Tocopherol & 1285.663673 & 0.785452 & -1285.427507 & 0.783243 & 146.85 \\
\hline
\end{tabular}

$\mathrm{SPE}_{\mathrm{p}}$, Single-point energies of parent molecule. $\mathrm{ZPVE}_{\mathrm{p}}$, Zero-point vibrational energies of parent molecule. $\mathrm{SPE}_{\mathrm{r}}$, Single-point energies of cation radical. $\mathrm{ZPVE}_{\mathrm{r}}$, Zero-point vibrational energies of cation radical

and found the $\alpha$-tocopherol's bond dissociation enthalpy value of $76.9 \mathrm{kcal} / \mathrm{mol}$ (Table-2).

Computational ionization potential data: Another important attribute of the effective antioxidant is the ionization potential that correlates electron-transfer mechanism via two steps reactions ${ }^{22}$;

$\mathrm{RO}_{2}{ }^{\wedge}+$ IRFIOH $(\mathrm{TxOH}) \rightarrow \mathrm{RO}_{2}^{-}+\mathrm{IRFIOH}^{+\wedge}\left(\mathrm{TxOH}^{+\wedge}\right)$

(electron transfer; radical cation formation)

$\mathrm{RO}_{2}^{-}+\mathrm{IRFIOH}^{+\wedge}\left(\mathrm{TxOH}^{+}\right) \rightarrow \mathrm{ROOH}+\mathrm{IRFIO}^{\wedge}(\mathrm{TxO})^{\wedge}$

(deprotonation)

In this way, the radical cation antioxidant produced by electron transfer and followed deprotonation in solution to give the corresponding radical. Low ionization potential values are favourable to raise the electron-transfer reactivity while high ionization potential decreases the electron-transfer rate between antioxidant and free radicals. Table- 3 shows the ionization potential values for IRFI, TxOH and $\alpha$-tocopherol. IRFI has lower ionization potential than $\mathrm{TxOH}$ and $\alpha$-tocopherol. Therefore, IRFI not only has lower bond dissociation enthalpy but also it has lower ionization potential than $\mathrm{TxOH}$ and $\alpha$-tocopherol.

Hydrogen atom transfer and/or electron transfer mechanism: Bond dissociation enthalpy is indicator for hydrogen atom transfer whereas electron transfer mechanism involves with ionization potential value for chain-breaking antioxidant activity. Wright et al..$^{23}$ proposed possibly hydrogen atom transfer mechanism rather than electron transfer for the radical scavenging effect of tocopherols based on the values of $\Delta \mathrm{IP}\left(\mathrm{IP}_{\text {compound }}-\mathrm{IP}_{\text {phenol }}\right)$ and $\Delta$ Bond dissociation enthalpy $\left(\mathrm{BDE}_{\text {compound }}-\mathrm{BDE}_{\text {phenol }}\right)$ values. They reported that up to $\triangle \mathrm{IP}=$ -36 and $\triangle \mathrm{BDE}=-10 \mathrm{kcal} / \mathrm{mol}$, radical scavenging activity is dominated by hydrogen atom transfer, but for $\Delta \mathrm{IP}>-36$, reactions tend to be predominantly electron transfer mecha$n^{n i s m}{ }^{23}$. There are some reports indicated that tocopherols scavenge free radicals by hydrogen atom transfer mechanism ${ }^{24,20}$ whereas other findings focused on the electron transfer mechanism ${ }^{25,26}$. Table- 4 shows that $\triangle \mathrm{IP}$ and $\triangle \mathrm{BDE}$ values for $\alpha$ tocopherol are -37.24 and $-10.42 \mathrm{kcal} / \mathrm{mol}$ respectively. Therefore, we believe both mechanisms (HAT and ET) are involved in the radical scavenging effect of tocopherol and this anitioxidant locates in the border regions of HAT/IP mechanism. This could be the reason of controversial reports about predominance of hydrogen atom transfer ${ }^{24}$ and electron transfer ${ }^{25,26}$ mechanism of $\alpha$-tocopherol in radical scavenging. While, IRFI has greater $\Delta \mathrm{IP}$ than tocopherol and $\mathrm{TxOH}$, so it can mainly react by electron transfer mechanism. Hence, Table- 4 clearly demonstrated that IRFI compound is a significantly efficient in radical scavenging via electron transfer mechanism because of having higher $\Delta \mathrm{IP}(-41.24 \mathrm{kcal} / \mathrm{mol})$. But, TxOH has lower $\Delta$ IP than $\alpha$-tocopherol. In contrast with IRFI, TxOH should be reacted with free radicals mainly via hydrogen atom transfer mechanism.

TABLE-4

CHANGE IN IONIZATION POTENTIAL ( $\triangle \mathrm{IPs}, \mathrm{kcal} / \mathrm{mol}$ ) AND BOND DISSOCIATION ENTHALPY ( $\triangle \mathrm{BDE}, \mathrm{kcal} / \mathrm{mol})$ OF TxOH, IRFI AND $\alpha$-TOCOPHEROL RELATIVE TO PHENOL

\begin{tabular}{lcc}
\hline Compounds & ${ }^{\mathrm{a}} \Delta \mathrm{BDE}$ & ${ }^{\mathrm{b}} \Delta \mathrm{IP}$ \\
\hline TxOH & -11.18 & -31.98 \\
IRFI & -12.28 & -41.24 \\
$\alpha$-Tocopherol & -10.42 & -37.24 \\
\hline a,b Relative to 87.32 and $184.09 \mathrm{kcal} / \mathrm{mol}$ for phenol in the same basis \\
set respectively
\end{tabular}

Finally, IRFI and $\mathrm{TxOH}$ have ionization potential that brings these molecules into the range where the electron transfer and hydrogen atom transfer mechanisms can become important respectively. But, both hydrogen atom transfer and electron transfer mechanisms are important in radical scavenging by $\alpha$-tocopherol.

Drug likeliness and bioavailability: Molecular properties and drug-likeness of IRFI, Trolox and $\alpha$-tocopherol molecules calculated via different tools in Table-5. Lipinski rule states, that most drug-like molecules have; octanol-water partition coefficient $(\log \mathrm{P}) \leq 5$, molecular weight $\leq 500$, $\mathrm{H}$-bond acceptors $\leq 10, \mathrm{H}$-bond donors $\leq 5$. Molecules violating more than one of these rules may have problems with bioavailability ${ }^{27,28}$. It was found that IRFI and $\mathrm{TxOH}$ satisfy the rule-of- 5 but $\alpha$-tocopherol violates one of the Lipinski rule $(\log \mathrm{P}>5) . \log \mathrm{P}$ is the main part of the rule-of-5 parameters that are widely applied in drug discovery to understand the 
absorption and permeability of compounds. $\log \mathrm{P}$ is a descriptor of the lipophilicity of a neutral substance that refers the octanol-water partition coefficient. Based on $\log \mathrm{P}$ values IRFI is more hydrophile and water soluble analogue of $\alpha$-tocopherol than $\mathrm{TxOH}$.

TABLE-5

MOLECULAR PROPERTIES AND DRUG-LIKENESS OF IRFI, TxOH AND $\alpha$-TOCOPHEROL

\begin{tabular}{|c|c|c|c|}
\hline *Properties & IRFI & $\mathrm{TxOH}$ & $\alpha$-Tocopherol \\
\hline${ }^{\mathrm{a}} \mathrm{MW}(\mathrm{g} / \mathrm{mol})$ & 236 & 250 & 430 \\
\hline a'H-Bond acceptors & 4.0 & 4.0 & 2.0 \\
\hline${ }^{\mathrm{a}} \mathrm{H}$-Bond donors & 2.0 & 2.0 & 1.0 \\
\hline apolar surface area $\mathrm{A}^{2}$ & 66.8 & 66.8 & 29.5 \\
\hline${ }^{\mathrm{a}} \log \mathrm{P}$ & 2.2 & 2.8 & 10.7 \\
\hline${ }^{\mathrm{b}} \log \mathrm{P}$ & 1.99 & 2.46 & 10.96 \\
\hline${ }^{c} \log \mathrm{P}$ & 2.33 & 3.37 & 8.67 \\
\hline${ }^{h} \log P$ & 2.92 & 3.46 & 9.83 \\
\hline$\mu^{\mathrm{g}}($ Debyes $)$ & 1.72 & 2.77 & 2.42 \\
\hline Lipinski violations & 0 & 0 & 1.0 \\
\hline $\begin{array}{l}\text { *The parameters were } \\
\text { broadinstitute.org/), }{ }^{\mathrm{b}} \mathrm{C} \\
{ }^{\mathrm{c}} \text { Molsoft (www.molsof } \\
\text { (using B3LYP/6-311 + }\end{array}$ & $\begin{array}{l}\text { led fr } \\
\text { pider } \\
\text { /mpro } \\
2 \mathrm{p})\end{array}$ & $\begin{array}{l}\text { hem ba } \\
\text { www.c } \\
\text { yperCh } \\
\text { et) }\end{array}$ & $\begin{array}{l}\text { ttp://chembank } \\
\text { pider.com), } \\
\text { hd }{ }^{\mathrm{g}} \text { Gaussian98 }\end{array}$ \\
\hline
\end{tabular}

Molecular dipole moment (the electric dipole moment of a molecule) can be defined as the product of magnitude of charge and distance of separation between the charges. Dipole moment ( $\mu$, in Debye) values refer the molecular geometry. Higher dipole moment correlated to the linearity (planarity) of molecule and higher antioxidant capability. For example, a planar catechin analogue indicated more efficient antioxidant activity than catechin ${ }^{29}$. Table-5 shows that the dipole moment for $\mathrm{TxOH}$ is larger than IRFI. We believe this property compensates the unfavourable important antioxidant parameters including bond dissociation enthalpy and ionization potential values for $\mathrm{TxOH}$ (Tables 2 and 3). Trolox has higher bond dissociation enthalpy and ionization potential than IRFI. Therefore, it is conceivable that IRFI should have higher antioxidant activity than trolox. Experimental findings are in contrast with theoretical bond dissociation enthalpy and ionization potential data, indicated similar antioxidant activity of TxOH with IRFI. Hence, higher linearity of $\mathrm{TxOH}$ causes $\mathrm{TxOH}$ would be more active than expected from computational bond dissociation enthalpy and ionization potential values.

\section{Conclusion}

The use of water-soluble vitamin E derivatives might be considered as a new important therapeutic tool in oxidative stressor conditions. Studying the antioxidant activity of TxOH as well as novel synthetic hydrophilic-derivative of vitamin E, IRFI, demonstrates that both $\mathrm{TxOH}$ and IRFI have similar antioxidant potency for extra-cellular and intra-cellular conditions. Both of TxOH and IRFI have cell membrane permeable effectiveness and powerful antioxidant activity in cellular environment with antitoxic ability. The efficient cellular protection of IRFI and $\mathrm{TxOH}$ against toxic free radicals can be explained by the capability of every one IRFI and TxOH to reduce two free radicals. But, their radical scavenging mechanisms are different. Trolox mainly acts as potent hydrogen atom transfer (HAT) whereas IRFI acts via electron transfer (ET) mechanism for radical chain-breaking reaction. In contrast to IRFI and $\mathrm{TxOH}$, both mechanisms are conceivable in radical scavenging by $\alpha$-tocopherol. In conclusion, IRFI has similar antioxidant activity with $\mathrm{TxOH}$ including cell-permeable, potent antioxidant, used in biological or biochemical applications to reduce oxidative stress or damage and can be applied instead of $\mathrm{TxOH}$ as a standard or positive control in antioxidant assays.

\section{ACKNOWLEDGEMENTS}

The authors gratefully acknowledged for the financial support provided by Research Institute for Fundamental Sciences, University of Tabriz and Iran National Science Foundation, Iran.

\section{REFERENCES}

1. M.G. Traber, Biofactors, 10, 115 (1999).

2. M.J. Fryer, Plant Cell Environ., 15, 381 (1992).

3. S. Lemaire-Ewing, S. Desrumaux, D. Néel and L. Lagrost, Mol. Nutr. Food Res., 54, 631 (2010).

4. R. Gajdocsi, A. Bikov, B. Antus, I. Horvath, P.J. Barnes and S.A. Kharitonov, J. Aerosol Med. Pulm. Drug. Deliv., 24, 271 (2011).

5. Y. Gole, O. Gargne, M. Coulange, J.G. Steinberg, M. Bouhaddi, Y. Jammes, J. Regnard and A. Boussuges, Eur. J. Appl. Phys., 111, 937 (2011).

6. L.A. Rowe, N. Degtyareva and P.W. Doetsch, Free Radic. Biol. Med., 45, 1167 (2008).

7. F. Distelmaier, H.J. Visch, J.A.M. Smeitink, E. Mayatepek, W.J.H. Koopman and P.H.G.M. Willems, J. Mol. Med. (Berl.), 87, 515 (2009).

8. A. Muñoz-Acevedo, L.Y.V. Méndez, E.E. Stashenko and V.V. Kouznetsov, Anal. Chem. Lett., 1, 86 (2011).

9. G.M. Campo, S. Ceccarelli, F. Squadrito, D. Altavilla, L. Dorigotti and A.P. Caputi, Drug Rev., 15, 157 (1997).

10. R. Carmelo,A. Pietro, E. Maria, M. Herbert, I. Pietro, T. Nunzio, A. Domenica, B. Alessandra, Z. Biagio and S. Francesco, Urol. Res., 32, 367 (2004).

11. A. Barzegar, N. Chaparzadeh, N. Zarghami, J.Z. Pedersen and S. Incerpi, J. Iran. Chem. Soc., 93, 1880 (2011).

12. A.Barzegar and A.A. Moosavi-Movahedi, PLoS One, 6, e26012 (2011).

13. A. Gomes, E. Fernandes and J.L.F.C. Lima, J. Biochem. Biophys. Methods, 65, 45 (2005)

14. G.A. DiLabio, D.A. Pratt and J.S. Wright, J. Org. Chem., 65, 2195 (2000).

15. M.J.S. Dewar, E.G. Zoebisch, E.F. Healy and J.J.P. Stewart, J. Am. Chem. Soc., 107, 3902 (1985).

16. G.A. DiLabio, D.A. Pratt and J.S. Wright, Chem. Phys. Lett., 311, 215 (1999).

17. M.J. Frisch, et al., Gaussian 98, Gaussian Inc: Pittsburgh, PA (1998).

18. H. Shi, N. Noguchi and E. Nik, Methods Enzymol., 335, 157 (2001).

19. T.S. Chan, N. Shangari, J.X. Wilson, H. Chan, R.F. Butterworth and P.J. O'Brien, Free Radic. Biol. Med., 38, 867 (2005).

20. M. Lucarini and G.F. Pedulli, J. Org. Chem., 59, 5063 (1994).

21. M.E.J. Coronel and A.J. Colussi, Int. J. Chem. Kinet., 20, 749 (1988).

22. S. Matsushita, in eds.: O. Hayaishi, E. Niki, M. Kondo and T. Yoshikawa, Antioxidant studies in retrospect, New York: Elsevier, vol. 1, pp. 327334 (1988)

23. J.S. Wright, E.R. Johnson and G.A. DiLabio, J. Am. Chem. Soc., 123, 1173 (2001).

24. D. Njus and P.M. Kelley, FEBS Lett., 284, 147 (1991).

25. K. Mukai, Y. Kageyama, T. Ishida and K. Fukuda, J. Org. Chem., 54, 552 (1989).

26. S. Nagaoka, A. Kuranaka, H. Tsuboi, U. Nagashima and K. Mukai, J. Phys. Chem., 96, 2754 (1992).

27. C.A. Lipinski, F. Lombardo, B.W. Dominy and P.J. Feeney, Adv. Drug Del. Rev., 23, 3 (1997).

28. C.A. Lipinski, J. Pharm. Toxicol. Methods, 44, 235 (2000).

29. K. Fukuhara, I. Nakanishi, H. Kansui, E. Sugiyama, M. Kimura, T. Shimada, S. Urano, K. Yamaguchi and N. Miyata, J. Am. Chem. Soc., 124, 5952 (2002). 